\title{
Improving riverbed sediment classification using backscatter and depth residual features of multi-beam echo-sounder systems
}

\author{
Dimitrios Eleftherakis, ${ }^{\text {a) }}$ AliReza Amiri-Simkooei, ${ }^{\text {b) }}$ Mirjam Snellen, and Dick G. Simons \\ Acoustic Remote Sensing Group, Faculty of Aerospace Engineering, Delft University of Technology, \\ Kluyverweg 1, 2629 HS Delft, The Netherlands
}

(Received 7 September 2011; revised 29 February 2012; accepted 29 February 2012)

\begin{abstract}
Riverbed and seafloor sediment classification using acoustic remote sensing techniques is of high interest due to their high coverage capabilities at limited cost. This contribution presents the results of riverbed sediment classification using multi-beam echo-sounder data based on an empirical method. Two data sets are considered, both taken at the Waal River, namely Sint Andries and Nijmegen. This work is a follow-up to the work carried out by Amiri-Simkooei et al. [J. Acoust. Soc. Am. 126(4), 1724-1738 (2009)]. The empirical method bases the classification on features of the backscatter strength and depth residuals. A principal component analysis is used to identify the most appropriate and informative features. Clustering is then applied to the principal components resulting from this set of features to assign a sediment class to each measurement. The results show that the backscatter strength features discriminate between different classes based on the sediment properties, whereas the depth residual features discriminate classes based on riverbed forms such as the "fixed layer" (stone having riprap structure) and riverbed ripples. Combination of these two sets of features is highly recommended because they provide complementary information on both the composition and the structure of the riverbed. (C) 2012 Acoustical Society of America.
\end{abstract}

[http://dx.doi.org/10.1121/1.3699206]

PACS number(s): 43.30.Pc [AIT]

Pages: $3710-3725$

\section{INTRODUCTION}

The morphology and sediment composition of the sea/ river bed is of high importance to a large number of offshore activities such as oil and gas exploration, the installation of offshore windmill farms, and the study of marine biology. Furthermore, in countries like the Netherlands, where a great number of rivers are used for navigation, insight into the river morphology and its dynamic behavior, and sediment composition is essential. An attractive system for obtaining information both for the sea/river bed bathymetry and sediment composition is the multi-beam echo-sounder (MBES). The sonar emits short pulses of sound towards the sea/river floor to determine the depth and the backscatter strength for a large number of beams. The MBES provides high spatial coverage of an area at moderate costs and within a short time. Therefore, it appears as a good alternative to the conventional, expensive and time-consuming approach of mapping the sea/river floor composition by taking a large number of physical sediment samples. A brief overview of the techniques used for determining sediment properties in shallow waters is given in Ref. 1.

The MBES classification methods can be divided into phenomenological (or empirical) and model-based (or physical). Model-based approaches make use of physical models and determine the sediments type by maximizing the match between modeled and measured signals or signal features,

\footnotetext{
${ }^{\text {a) }}$ Author to whom correspondence should be addressed. Electronic mail: d.eleftherakis@tudelft.nl

b) Also at: Department of Surveying Engineering, Faculty of Engineering, University of Isfahan, Isfahan 81746-73441, Iran.
}

where sediments type or parameters indicative of sediment floor type, are input into the model. These approaches allow for direct coupling between the acoustic classes and sediment characteristics if the MBES sensitivity is known. On the other hand, the empirical approaches base the classification on features of the data, after dividing the area into small regions. This approach is considered in the present work. The outcome of this approach is a qualitative description of the sediment distribution of an area (e.g., finer, fine, coarse, coarser), but ground truth is required for associating the classification results to physical parameters of the sediments (e.g., mean grain size). The advantage of the empirical methods is their ease of implementation and use.

The main information provided by an MBES is the backscatter strength and the bathymetry of the area. Both can be treated as individual values or as images by plotting their spatial distributions over an area. ${ }^{2}$ The potential of the backscatter for empirical sediment characterization has been highlighted in previous work. ${ }^{3,4}$ Significant work in this field has been done by Quester Tangent Corporation (QTC) where 132 features are calculated and processed both from the backscatter amplitude and backscatter texture. Detailed information about QTC can be found in Refs. 5-11. During the last few years, research considered the potential of the information contained in the bathymetry for classification ${ }^{12,13}$ and now the interest has focused on how to combine a broader range of information that may include backscatter and bathymetry features in many forms into the clustering models. ${ }^{14}$ An extensive review of the different classification approaches can be found in Ref. 15.

In two previous studies ${ }^{16,17}$ a new model-based method employing backscatter, denoted as the Bayesian classification 
methodology (BCM), for deriving the sediment distribution in two parts (Sint Andries and Nijmegen) of the River Waal in the Netherland gave promising results. However, one important artificial riverbed feature, the fixed layer, was not identified by the method as a separate bottom type. This fixed layer consists of big stones with rip rap structure and is applied for fixation of the sediment. In this paper a combination of backscatter and a bathymetric feature, namely the residuals of depth, is successfully used to identify the different sediment classes, including the fixed layer. Principal components analysis (PCA) is used for data reduction and the common $\mathrm{K}$-means method for clustering the data. The importance of the present paper is twofold: (1) it clearly demonstrates the advantage of the combination of features on capturing the range of different formations on riverbeds, and (2) it provides a physical explanation of the contribution of each feature.

The paper is organized as follows. Section II gives information both about the surveyed areas and the details of the surveys. This section also provides a brief description of the methodology of Refs. 16 and 17, and results obtained from the previous studies on the same areas. Section III provides details on the methodology (PCA and K-means clustering) used for identifying the different sediment types. Section IV describes the data preparation procedures. Section V presents and discusses the results from the PCA and K-means clustering analysis. Finally, the main conclusions are summarized in Sec. VI.

\section{EXPERIMENTS AND PREVIOUS RESULTS}

\section{A. A description of the surveyed areas}

The MBES data was collected from parts of the Waal River in the Netherlands during surveys conducted by the Directorate General for public works and water management. The Waal River is one of the branches of the Rhine River and the main inland waterway transport artery between the port of Rotterdam and urban and industrial areas of Germany. The Waal River has prominent bends at Nijmegen and Sint Andries. The interplay of water and sediment in these bends had produced characteristic bed topography of deep outer-bend pools and shallow inner-bend point bars. The latter formed obstacles for navigation, despite the large depth available in the pools, because they reduced the space for two-way traffic with possibilities to overtake other ships. The problem has been addressed using three strategies: (1) by dredging in order to remove the shoals, (2) by constructing groynes in order to change the alignment of the river, and (3) by constructing non-erodible layers in order to lower the point bar and increase the navigation width. Therefore, the deep pools have been filled and covered with riprap to form a fixed, non-erodible layer in the outer bend. The resulting scour in the inner-bend made the river sufficiently deep over a larger width.

The first fixed layer is located between Sint Andries and Zuilichem ( $\mathrm{km} \mathrm{925-928).} \mathrm{It} \mathrm{is} 140 \mathrm{~m}$ wide and $3.1 \mathrm{~km}$ long, and was constructed in the years 1997-1999. It lies $3.5 \mathrm{~m}$ below the Dutch river low water reference level (OLR). ${ }^{18}$ The bathymetry (not referenced to OLR) of the river at Sint Andries as produced from an October 2007 multi-beam survey is presented in Fig. 1 (top). It shows the general pattern of shallow inner bends and deeper outer bends, but also the more detailed forms of underwater dunes as well as local scour holes at the tips of transverse river training structures called "groynes."

The second fixed layer is located in the Waal river bend at Nijmegen $(\mathrm{km} \mathrm{883-885).} \mathrm{This} \mathrm{fixed} \mathrm{layer} \mathrm{has} \mathrm{a} \mathrm{width} \mathrm{of}$ $150 \mathrm{~m}$, a length of $2 \mathrm{~km}$ and a depth of $3.5 \mathrm{~m}$ below the OLR. ${ }^{18}$ It was constructed in the years 1986-1988. Figure 1 (bottom) presents the bathymetry of the river in this area as produced from a May 2008 multi-beam survey, again not referenced to OLR.

Samples were collected from both areas, using a bottom grab. Figure 2 shows the histograms of the distribution of the mean grain size of the samples collected at Sint Andries (Fig. 2, left) and at Nijmegen (Fig. 2, right).

\section{B. Details of the surveys}

The sonars used in both surveys were of the same type: a Kongsberg EM3002 single-head multi-beam echo-sounder. This sonar is well suited for shallow water depths as its high frequency ensures narrow beams with small physical dimensions. The operational frequency was $300 \mathrm{kHz}$ and the maximum number of beams (of equidistant pattern) per ping was 254. The swath width was $130^{\circ}$, the pulse length $150 \mu \mathrm{s}$, and the maximum ping rate $40 \mathrm{~Hz}$. The beam width was $1.5^{\circ} \times 1.5^{\circ}$ at nadir. All beams were electronically stabilized for pitch and roll. For each beam and each ping a single backscatter value is given. This value is the result of first applying a moving average over the time series of amplitude values and then selecting the maximum average level of each beam. ${ }^{19}$

\section{A summary of applying the $\mathrm{BCM}$ to the areas}

The method in Refs.16, 17, and 20 employs the backscatter strength collected at a certain incident angle instead of studying the angular behavior of the backscatter strength. The classification is performed per angle separately from other angles and hence is considered to be angle-independent. The method is based on the assumption that the backscatter values are an average value of the sample amplitude values. Therefore, according to the central limit theorem-for independent random values - the averaged backscatter value for a single sediment type follows a Gaussian distribution for a sufficiently large number of scatter pixels. Figure 3 illustrates this principle. In this figure $\theta$ is the grazing angle and $\varphi$ the angle of incidence. The required large number of scatter pixels is achieved for shallow waters ${ }^{16}$ by averaging the backscatter values over small surface patches, which consist of a small number of beams in the across-track direction and a few pings in the along-track direction. The creation of surface patches, apart from ensuring Gaussianity, has the additional advantage that it allows for two kinds of corrections of the backscatter data due to the presence of slopes: one correction to account for changes of the signal footprint's area, and a second correction to account for the slope effect on the beam grazing angle.

The method fits a number of Gaussian Probability Density Functions (PDFs) to the histogram of the backscatter 

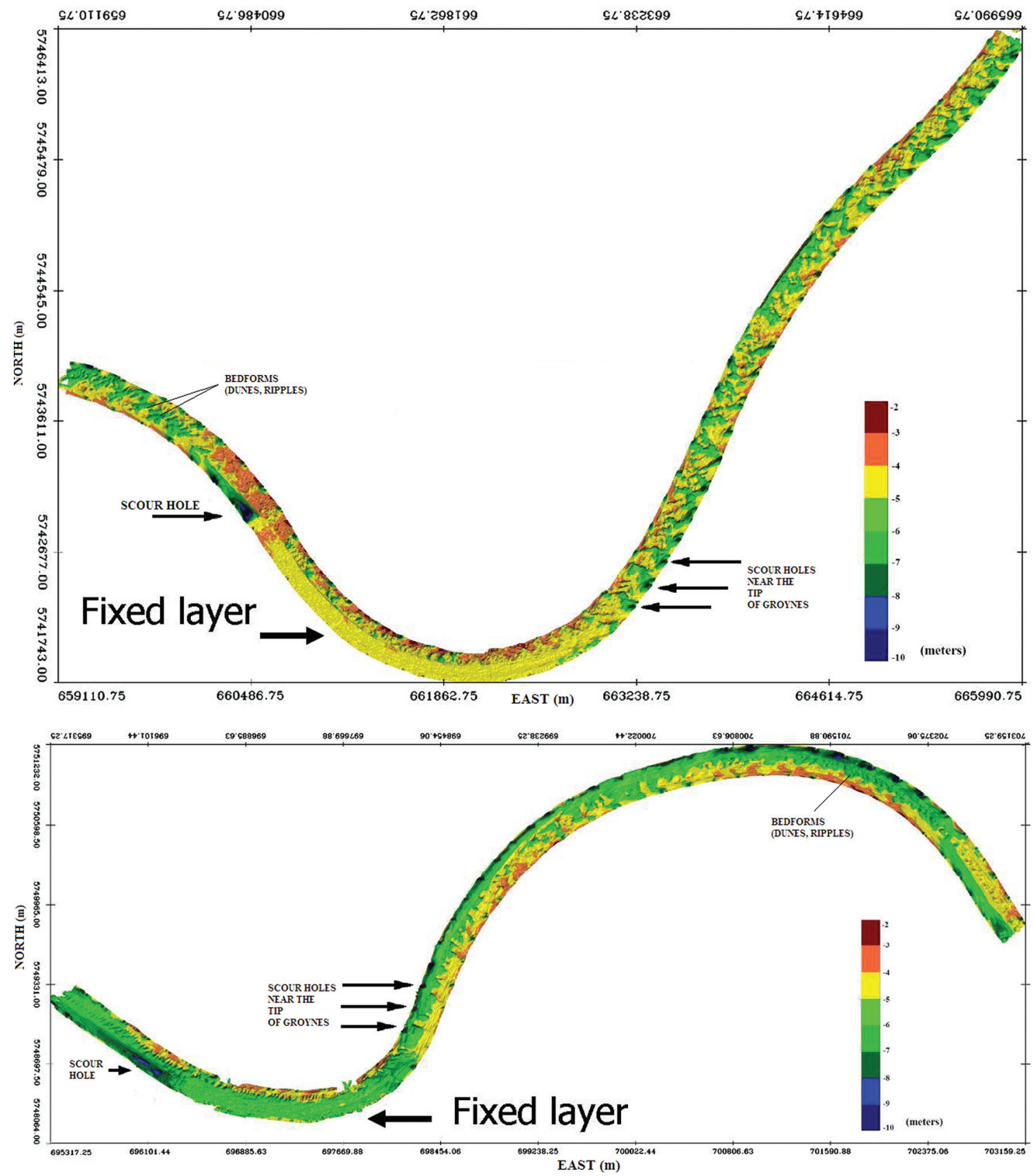

FIG. 1. Bathymetry of the Waal River at (top) Sint Andries and (bottom) Nijmegen. Indicative positions of scour holes behind the fixed layer, scour holes at the tips of groynes, and bed foms (ripples and dunes) are shown in the figure.

data at a given incident angle. The optimum number of PDFs is found by consecutively increasing the number of PDFs until a chi-square distributed test statistic becomes less than a critical value. The number of Gaussians then represents the maximum number of classes that can be discriminated based upon the backscatter values, and the borders of the classes are the intersections of each Gaussian with its neighbor. The backscatter data at a few low grazing angles are processed in order to estimate the mean backscatter strength, the variance and its coefficient per class. These parameters are used as guidance for the statistical processing of all other angles. The surface patches in Refs. 16 and 17 were of size $0.5 \mathrm{~m} \times 0.5$ $\mathrm{m}$, but the final maps resulted after using weighted moving average for surface patches of $2 \mathrm{~m} \times 2 \mathrm{~m}$.
Three classes were identified for each area. The plots can be seen in Refs.16 and 17. The areas of the fixed layer could not be discerned from the other parts of the river.

\section{METHODOLOGY}

In this section we briefly explain basic concepts of the PCA and the K-means clustering approach. Though the methods are well established, many variations of them exist in the literature, depending on the nature of different investigations. Therefore, the scope of this section is to provide only the necessary details of the methodology followed in this paper for creating the sediment distribution maps of the Sint Andries and Nijmegen areas. 

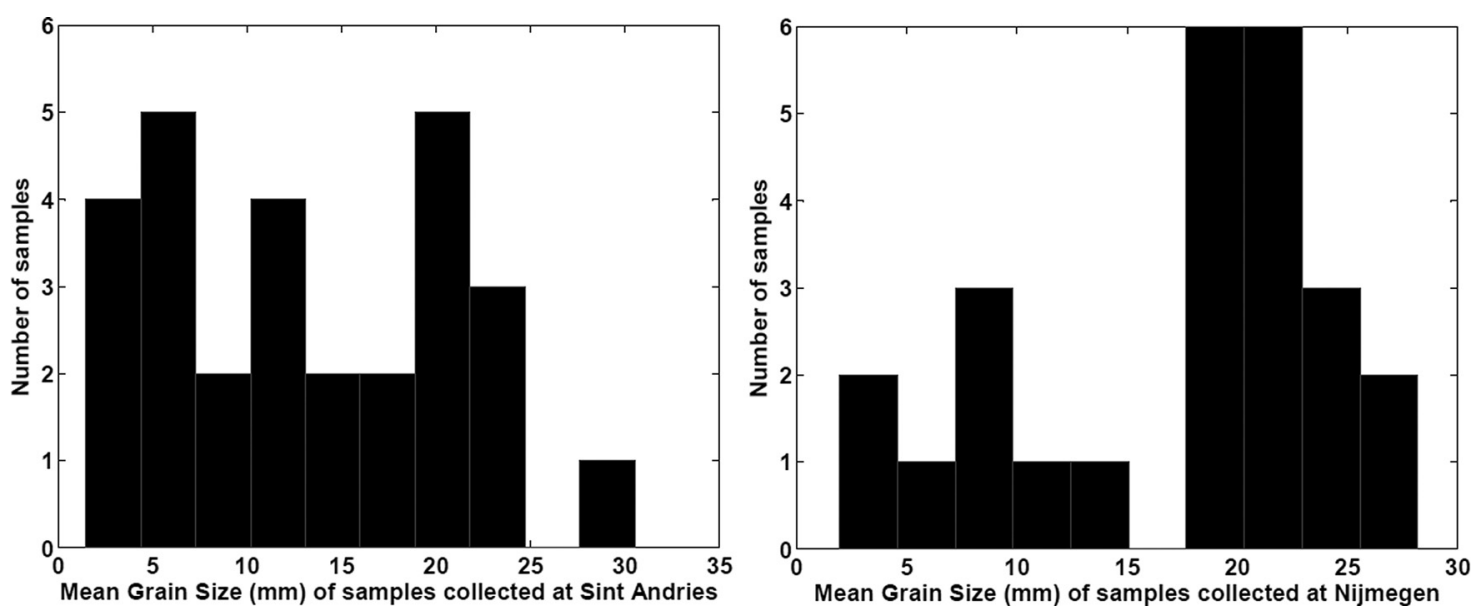

FIG. 2. Histograms of the mean grain size distribution of the samples collected (left) at Sint Andries and (right) at Nijmegen.

\section{A. Principal component analysis}

The PCA was first described by Refs. 21 and 22. The aim of the PCA is to reduce a multivariate dataset into a small set of variables called principal components (PCs) that can adequately describe the variability of the whole dataset. The reduction can be achieved if the original variables are at least moderately correlated. ${ }^{23}$ Unlike the initial features, the PCs are independent, thus each PC represents a different dimension in the data. Furthermore, they are sorted in descending importance order from the first PC, which explains the largest amount of the data variation to the last one representing the smallest variation. Therefore, $\operatorname{var}\left(Y_{1}\right) \geq \operatorname{var}\left(Y_{2}\right) \geq \cdots$ $\geq \operatorname{var}\left(Y_{p}\right)$, where $Y_{i}$ is the $i$ th principal component.

Considering the (nxp) matrix $F$ containing all $n$ measurements of the $p$ features, a principal component $Y_{i}$ is a linear combination of the $p$ original features (variables) $F_{1}, F_{2}, \ldots, F_{p}\left(F_{i}\right.$ denoting the $i$ th column of $\left.F\right)$ as

$$
Y_{i}=a_{i 1} F_{1}+a_{i 2} F_{2}+\ldots+a_{i p} F_{p}
$$

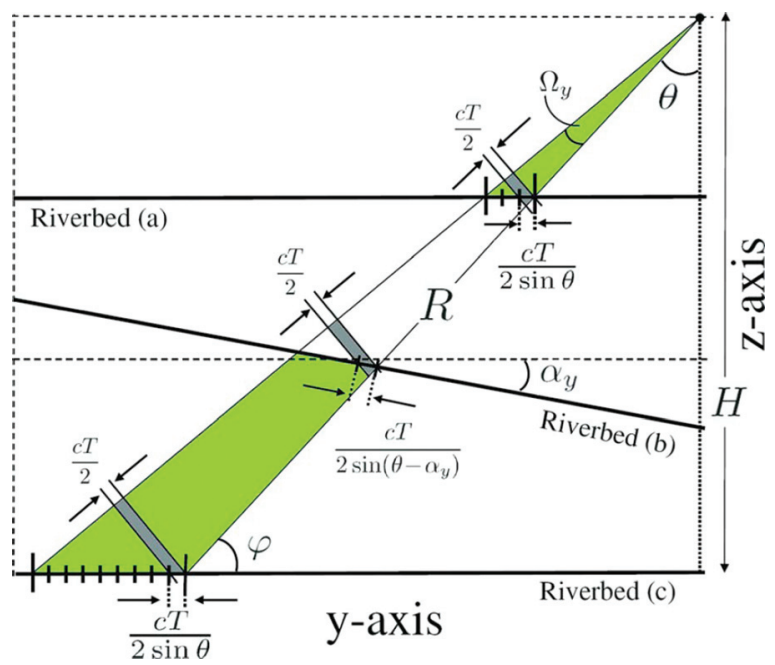

FIG. 3. Across-track cross section (y-z plane) for signal footprint of an oblique beam for three configurations: (a) shallow water, (b) non-flat bottom, and (c) deep water. $\theta$ denotes the grazing angle and $\varphi$ the incident angle. The figure has been taken from Ref. 16. with the condition that its variance, $\operatorname{var}\left(Y_{i}\right)$, is maximum, subject to the constraint that ${ }^{23} a_{i 1}^{2}+a_{i 2}^{2}+\ldots+a_{i p}^{2}=1$, and that $Y_{i}$ is uncorrelated to the other principal components, thus having zero covariance, i.e., $\operatorname{cov}\left(Y_{i}, Y_{k}\right)=0$ for $i \neq k$. The calculation of the principal components is performed in four steps:

First, the original features $f_{j i}$, where $i=1,2, \ldots, p$ (number of features) and $j=1,2, \ldots, n$ (size of data) are standardized. This step is necessary when the variables have different scales or common scale with significantly different ranges. The standardization is performed as follows: ${ }^{24}$

$$
z_{j i}=\frac{f_{j i}-\mu_{f_{i}}}{\sigma_{f_{i}}},
$$

where $\mu_{f_{i}}$ and $\sigma_{f_{i}}$ are the sample mean and standard deviation of the feature $i$, respectively,

Second, the covariance matrix $R$ of the standardized data $Z$ is determined as ${ }^{24}$

$$
R=\frac{Z^{T} Z}{n-1}
$$

where $Z=\left[\begin{array}{cccc}z_{11} & z_{12} & \cdots & z_{1 p} \\ z_{21} & z_{22} & \cdots & z_{2 p} \\ \vdots & \vdots & \ddots & \vdots \\ z_{n 1} & z_{n 2} & \cdots & z_{n p}\end{array}\right]$.

The diagonal elements of $R$ are the variances of the features, all having a value of 1 due to standardization, while the non-diagonal elements of $R$ are the correlation coefficients among the features.

Third, the eigenvalue decomposition of the matrix $R$ is obtained as

$$
R=E \Lambda E^{T},
$$

where $E$ is the square matrix of the eigenvectors of $R$ and $\Lambda$ is the diagonal matrix of the corresponding eigenvalues (each column of $E$ corresponds to one element of $\Lambda$ ).

Finally, the PC matrix $Y$ is calculated by multiplying the original data matrix $F$ with the eigenvectors matrix $E$ as

$$
Y=F E \text {. }
$$


After obtaining the $Y$ matrix, the optimum subset of PCs has to be determined. The subset must consist of the minimum number of PCs that contain most of the original data's information. A large number of tools exist for deciding the optimum number of PCs. An extensive comparison between various tools can be found in Ref. 25.

In the current research we consider one of the simplest but still acceptable tools ${ }^{26}$ available for selecting the number of principal components. The criterion is to choose adequate PCs to explain a specific percentage of the total variability in the data. The percentage of the standardized data variance due to the first $m(m \leq p)$ PCs is given as

$$
t_{m}=100 \times \frac{\sum_{k=1}^{m} \lambda_{k}}{\sum_{k=1}^{p} \lambda_{k}},
$$

where $\lambda$ is the variance explained by each principal component. The threshold percentage varies $(70 \%-90 \%)^{26}$ and frequently depends on the specific details of a data set.

Our application of PCA in this contribution is twofold: (1) PCA was used to determine the most appropriate features among all features, thus reducing the number of the original variables to only the necessary ones, and (2) the optimum set of PCs are grouped into different classes by using K-means clustering.

\section{B. K-means clustering}

The K-means ${ }^{27}$ unsupervised algorithm partitions $n$ observations into $k$ mutually exclusive subsets $S_{\mathrm{j}}$ (clusters) so as to minimize the sum of point to centroid (point whose parameter values are the average of the parameter values of all points in a cluster) squared Euclidean distances:

$$
J=\sum_{j=1}^{k} \sum_{n \in S_{j}}\left|x_{n}-\mu_{j}\right|^{2},
$$

where $x_{n}$ is the $n$th data point and $\mu_{j}$ is the geometric centroid of the data points in $S_{j}$. Detailed information about the algorithm of the K-means clustering can be found in Ref. 28.

In this paper, two tools are considered in conjunction for determining whether the preselected number of clusters is acceptable or not: (1) the total sum of distances, and (2) the silhouette plot.

The total sum of distances has to decrease for successive ascending values of $k$ in order to successfully partition the dataset into clusters. However, this sum always decreases with an increasing number of clusters. Therefore, we have selected the relative reduction in the sum, expressed as the percentage reduction. A second tool for determining the quality of the separation between clusters is the silhouette plot, which is mainly a visual measure. One may also define the silhouette coefficient for an individual point as ${ }^{29}$

$$
s_{i}=\frac{b_{i}-a_{i}}{\max \left(a_{i}, b_{i}\right)},
$$

where $a_{i}$ is the average distance (dissimilarity) of the point $i$ to all other points of its cluster, and $b_{i}$ gives the minimum average distance (dissimilarity) of $i$ to all points of other clusters. Values for $s_{i}$ for each cluster fall in the range from -1 to 1 . A negative value is undesirable because it corresponds to the case that $a_{i}$ is greater than $b_{i}$. A desirable case occurs, in general, when the silhouette coefficient is positive indicating that $a_{i}<b_{i}$, and, in particular, when it is close to one. Therefore, $s_{i}=1$ indicates that there is a high probability for the points to be successfully clustered. The average silhouette coefficient (aSC) of all the clusters can be used as a quantitative criterion. A proposed (see Table I) interpretation is described in Ref. 29.

In general, a definite selection of an optimal number of clusters is ambiguous. Therefore, in this paper the number of clusters in most of the cases is predefined based on the knowledge gained from Refs. 16 and 17 and the two tools described above are used to determine whether the separation of the selected number of clusters is within the acceptable range or not.

\section{DATA PREPARATION}

\section{A. Extracting the features from the surface patches}

The data were grouped per beam, in small surface patches, where each surface patch consisted of the mean data values over a few beams in the across-track direction and a few pings in the along-track direction. The typical size of each surface patch was approximately $0.5 \mathrm{~m} \times 0.5 \mathrm{~m}$ and all the features were determined for this surface size. This procedure was the same data grouping procedure followed in Refs. 16 and 17. For this paper, the data size was, at the last stage, further reduced by constructing boxes of $10 \mathrm{~m} \times 10 \mathrm{~m}$ in order to be able to process effectively the whole dataset and significantly reduce the fluctuations of the features extracted. For example, the standard deviation of the residuals was calculated for each one of the $0.5 \mathrm{~m} \times 0.5 \mathrm{~m}$ boxes and then these values were averaged over $10 \mathrm{~m} \times 10 \mathrm{~m}$ boxes to give the final values of the standard deviation of the residuals. For the approach of Refs. 16 and 17 the larger boxes could not be made, because it would result in combining backscatter values over a range interval where the angle dependence can not be neglected.

For each surface patch the average, the standard deviation, and higher-order statistical moments were determined for both the backscatter strength and the least-squares depth residuals. In addition, the slopes of the surface patches were considered. The backscatter value is an important classification parameter of the sediments on river/sea beds ${ }^{3,4}$ and should be accounted for in the classification. The residuals

TABLE I. Proposed interpretation of the average Silhouette Coefficient (aSC) (Ref. 29).

\begin{tabular}{lc}
\hline \hline aSC & Proposed Interpretation \\
\hline $0.71-1.00$ & A strong structure has been found \\
$0.51-0.70$ & A reasonable structure has been found \\
$0.26-0.50$ & The structure is weak and could be artificial; \\
& please try additional methods on this data set \\
$<0.26$ & No substantial structure has been found \\
\hline \hline
\end{tabular}


represent the difference between the observed depth values and the fitted surface patch estimated by the least squares method. In fact, they contain information about the sediments size variations in a "purer" way than the depth itself because they take the slopes of the patches into account.

A comment on the estimation of the least squares (LS) depth residuals is in order. The along-track $(x)$ and acrosstrack $(y)$ slopes of each surface patch were calculated based on the method described in Ref. 16. The polynomial used for fitting a surface to each patch has the form:

$$
z=f(x, y)=a_{o}+a_{1} x+a_{2} y+a_{3} x^{2}+a_{4} y^{2}+a_{5} x y .
$$

The LS method, details of which can be found in Ref. 30, was used to solve the over-determined system of equations. According to LS, the estimate of a linear model $E(z)=A a$ is determined as $\hat{a}=\left(A^{T} Q_{z}^{-1} A\right)^{-1} A^{T} Q_{z}^{-1} z$, where $A$ is the design matrix, $z$ is the vector of depth measurements, and $Q_{z}$ is the covariance matrix of $z$. The residual vector is calculated as $\hat{e}=A \hat{a}-z$.

The first four statistical moments, namely, mean, standard deviation, skewness, and flatness (kurtosis) of the backscatter and depth residuals were computed. The arithmetic mean is the most widely used statistical parameter. The standard deviation is also an important parameter since it gives a measure of the variability of the data. Skewness is a measure of the asymmetry of the distribution. Kurtosis is the measure of flatness of the data relative to a normal distribution. Skewness and kurtosis were selected because the $\mathrm{K}$-distribution is a potential sediment classification tool based on results from previous research ${ }^{16}$ as it can describe the backscatter distribution.

In addition, the median, mode, minimum, maximum, and mean absolute values were used as new features. In cases where the distribution of the backscatter and/or the residuals is not purely symmetric, the median is different from the mean, and can provide the middle point of the distribution. Mode is the value that has the most frequent occurrence within the data set. It shows the main tendency of the features within a surface patch. The minimum and maximum values were included as indicators of the data extremes.

For the residuals of the bathymetry, the mean absolute error (MAE) was calculated instead of the mean. Since the values of the residuals are small and can be positive and negative, the mean value could be each time close to zero. The mean absolute error is an average value for the absolute errors, given by the equation MAE $=\frac{1}{n} \sum\left|e_{i}\right|$. Therefore, it provides a measure of closeness between the predicted and measured values.

The 17 features that were taken into account for classification are summarized in Table II.

\section{B. Correcting for slope and angular effects in backscatter data}

Standard corrections to account for slopes were applied. In the present paper the same procedure and equations as those described in Ref. 16 were used for applying the corrections.
TABLE II. Features calculated for surface patches including statistical moments of backscatter values and least-squares depth residuals (16 features). The 17th feature is the total slope of each surface patch.

\begin{tabular}{lcccc}
\hline \hline & $\#$ & BS & $\#$ & LS depth residuals \\
\hline Mean (MAE) & 1 & $\sqrt{ }$ & 9 & $\sqrt{ }$ \\
Std. dev. & 2 & $\sqrt{ }$ & 10 & $\sqrt{ }$ \\
Skewness & 3 & $\sqrt{ }$ & 11 & $\sqrt{ }$ \\
Kurtosis & 4 & $\sqrt{ }$ & 12 & $\sqrt{ }$ \\
Median & 5 & $\sqrt{ }$ & 13 & $\sqrt{ }$ \\
Mode & 6 & $\sqrt{ }$ & 14 & $\sqrt{ }$ \\
Minimum & 7 & $\sqrt{ }$ & 15 & SLOPES \\
Maximum & 8 & $\sqrt{ }$ & 16 & 17 \\
\end{tabular}

Standardisation of the data was applied to account for the angular effect on the backscatter strength, as in performing the K-means clustering method, the data from all angles had to be gathered and processed at the same time. In fact, also the statistical distributions of the backscatter data are angular dependent. To account for this, the data of each angle was first standardised according to Eq. (2), allowing simultaneously processing the data corresponding to angles from $20^{\circ}$ to $70^{\circ}$. This standardization concerns the first and second statistical moments. Standardization of the higherorder moments might also be applied.

The effect of the standardization procedure followed in this paper can be seen in Fig. 4. The backscatter values versus angles for the complete dataset of Nijmegen have been plotted before standardization (Fig. 4, left), and after standardization (Fig. 4, right). Figure 4 (left) shows that there is an angular dependence of the backscatter values. This dependence is eliminated after standardization, resulting in the mean value of the backscatter measurements for all angles to be zero and the standard deviation to be one (Fig. 4, right). The same was done for Sint Andries.

\section{Determining the optimum set of features}

An important step before the classification is to determine those features among the 17 features presented in Table II that are representative of the river floor sediment. PCA was applied to the available features and the first 3 principal components that expressed most of the variability of the data (around $75 \%$ ) were obtained using Eq. (6). The features were then correlated to these principal components.

Figure 5 presents the results obtained following this strategy for Sint Andries (Fig. 5, left) and Nijmegen (Fig. 5, right) for all the 17 features. From these, it can be seen that the most informative features include the mean, median, minimum and mode of the backscatter and the mean absolute error, standard deviation, minimum and maximum of the least-squares depth residuals (Table III). This approach where the number of features is decreased, in this case from 17 to 8 , is a relatively standard approach and a variation of it is described in Ref. 26. It, in general reduces the number of PCs needed and consequently eases the interpretation of the results. In fact, using only one feature from each of the two parameters, i.e., reducing the number of features to 2 , is 

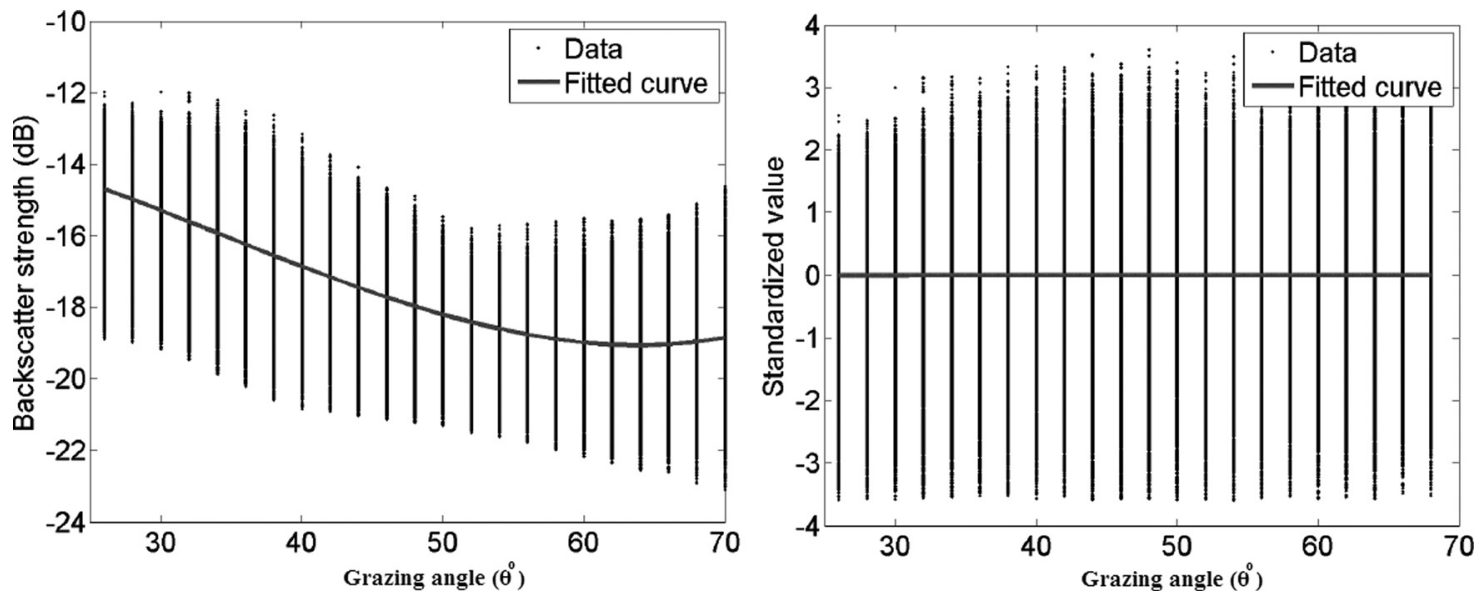

FIG. 4. Backscatter values versus grazing angles for Nijmegen (left) before standardization and (right) after standardization.

found to result in the same map as when using more features (see Sec. V). The balance between the two different sources (backscatter and LS residuals of depth) of information about the sediments was considered to be the optimum for providing reliable and highly discriminative classification results. It has to be noted that the threshold value has been selected in both cases as the value that satisfies three conditions: (1) it is close to the mean value of the ratio of correlations, (2) a sufficient number of features is included for the analysis (in this case 50\%), and (3) gives consistent results for both areas.

\section{RESULTS AND DISCUSSIONS}

This section is divided into four subsections. The first subsection presents the classification results for the two areas using the PCA and K-means clustering applied to the four extracted features of the backscatter strength. The second subsection presents the classification results based on the LS depth residual features. The third subsection gives the results based on all of the features extracted using the given backscatter and bathymetry data (Table III). Finally, the last subsection presents a discussion of the results.

\section{A. Classification based on backscatter strength}

The first investigation is to use the four backscatter features only and apply the PCA and K-means clustering to create classification maps of these two areas with three different sediment classes. We hence aim to directly compare the results of the Bayesian classification methodology ${ }^{16}$ and the K-means clustering method.

The results in Sint Andries area indicate that the first and second principal components account for $96.4 \%$ and $2.4 \%$ [using Eq. (6)] of the variability of the data, respectively. These numbers are $96 \%$ and $2.5 \%$ for Nijmegen. Because the first PC carries most of the variability, it is an indication for the presence of high correlation among the four backscatter features. Therefore, if one tries to obtain the classification map based on each of these features separately the results should be very similar.

The first two PCs are fed to the K-means clustering method. As a first step the clustering is carried out for three clusters equal to the number of sediment types that could be discriminated with the BCM. Figure 6 shows the scatter plots of the first PC versus the second PC of the clustered data. We can see that the "cuts" between the clusters are more or less parallel to the second PC. This explains that the first PC
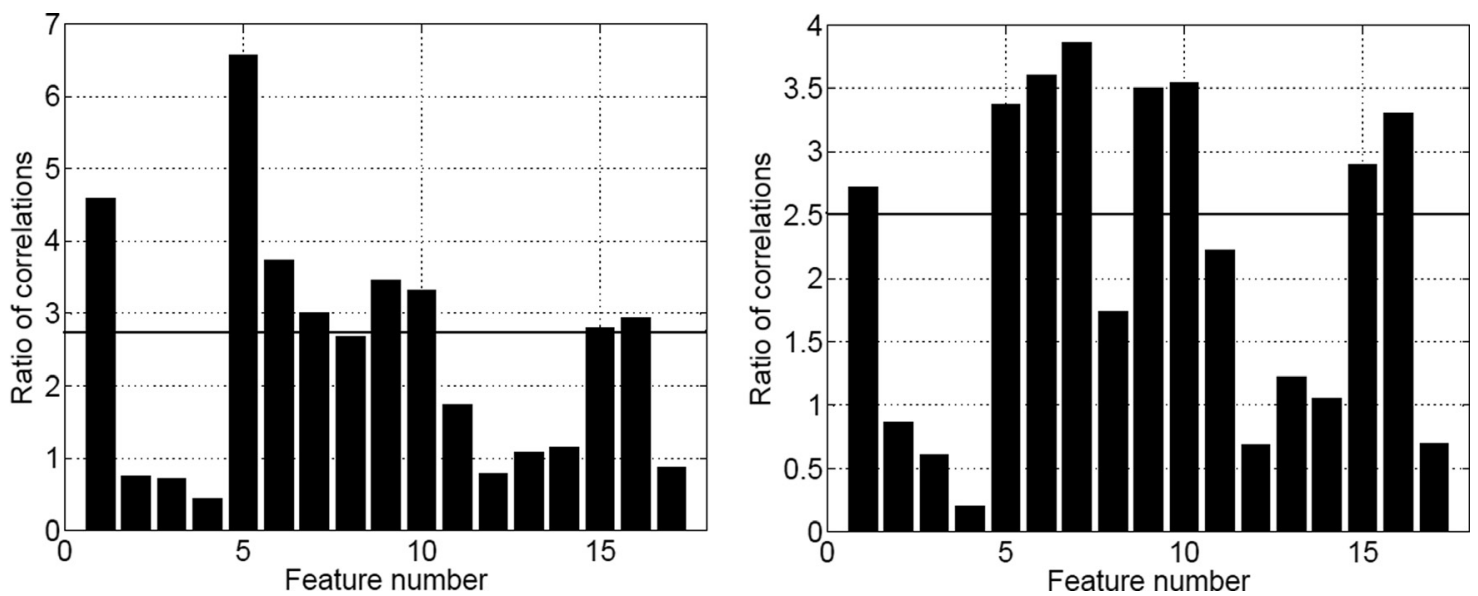

FIG. 5. Ratio of sum of absolute correlations for the first 3 principal components to sum of absolute correlations for the remaining components; (left) Sint Andries and (right) Nijmegen. 
TABLE III. Final remaining features; four backscatter features and four LS depth residuals features.

\begin{tabular}{lcc}
\hline \hline \# of feature & Backscatter & LS depth residuals \\
\hline 1 & Mean & \\
2 & Median & \\
3 & Minimum & \\
4 & Mode & \\
5 & & Mean absolute error \\
6 & & Standard deviation \\
7 & & Minimum \\
8 & & Maximum \\
\hline \hline
\end{tabular}

has the largest contribution to the clustering, and that the second PC has no significant effect; very similar results can thus be obtained using only the first PC. This supports our remarks made above about the significant correlation among the features.

In general empirical classification approaches provide acoustic classes only. For the situation considered here however the relation between the PCs and a physical parameter, namely backscatter, can be established. Based on this relation, colors have been added to Fig. 6, indicating higher backscatter values for lower values of the first PC. Here, green corresponds to fine material (low BS), yellow to intermediate (intermediate BS), and red to coarse (higher BS). This is based on the fact that highest values of the backscatter strength are expected for coarsest grains whereas as the grains become finer as the backscatter strength decreases.

The correspondent sediment distribution maps of Sint Andries and Nijmegen are shown in Fig. 7. In the Sint Andries area, the percentage of each class that resulted from the classification procedure is $28.9 \%, 42.2 \%$, and $28.9 \%$ for the fine, intermediate, and coarse sediments, respectively. These numbers change to $12.8 \% 43.4 \% 43.8 \%$ for Nijmegen. The percentages are different from those obtained using the Bayesian method in Refs. 16 and 17, which were 5\% (fine), $40 \%$ (intermediate), and 55\% (coarse) for Sint Andries, and $5 \%$ (fine), 30\% (intermediate), and 65\% (coarse) for Nijmegen. An important difference between the two methods is the number of measurements over which averaging is performed. For the approach of Refs. 16 and 17 the number of beams which can be averaged is limited due to the angular dependence of the backscatter data. In Ref. 16 a significant overlap existed between the three classes. Here due to the standardization, averaging was carried out over a much larger number of measurements, improving the discrimination performance.

As a second step, it is investigated whether it is possible to further increase the number of clusters. To this end, Fig. 8 presents the \% reduction (left $y$-axis) and the average silhouette coefficient (right $y$-axis) against the number of clusters from 2 to 8 for Sint Andries (Fig. 8, left) and Nijmegen (Fig. 8 , right). It is observed that the maximum $\%$ reduction in distances and the largest average silhouette coefficient is achieved for the case of 2 clusters but the value of aSC is still high for 3 clusters (0.66 and 0.63 for Sint Andries and Nijmegen, respectively) showing that the separation of the 3 clusters is "almost strong" according to Table I. When increasing the number of clusters to more than 3 , the two parameters gradually drop, indicating that further discrimination will be accompanied with less good separated clusters. Therefore, for the current paper the investigation stops at $k=3$.

\section{B. Classification based on LS depth residuals}

The first step is to determine a suitable number of classes that could be obtained by using the four statistical features determined from the LS depth residuals. In Sint Andries, application of the PCA indicates that the first and second PCs account for $99.7 \%$ and $0.2 \%$ of the variability of the data, respectively. These numbers are $99.4 \%$ and $0.38 \%$ for Nijmegen. Again it can be concluded that high correlation exists among the four features. Figure 9 shows the combined plot of the \% reduction in the distances (left $y$-axis) and the average silhouette coefficient (right $y$-axis) against the number of clusters for Sint Andries (Fig. 9, left) and Nijmegen (Fig. 9, right). It is apparent that the case of 4 clusters appears to provide good separated clusters since both the values of the \% reduction and the aSC are high (more than 0.7). Therefore, the suitable number of clusters was selected as 4 .

For the clustered data, again a "parallel cut" to the second PC appears, indicating that if one uses only the first PC

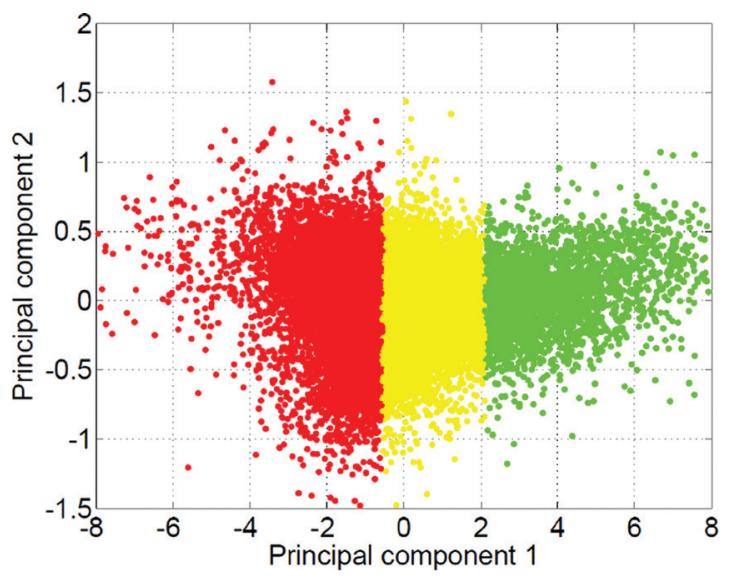

FIG. 6. Scatter plot of PC1 versus PC2. The colors indicate different clusters resulting from four backscatter features $(k=3)$; (left) Sint Andries and (right) Nijmegen. 

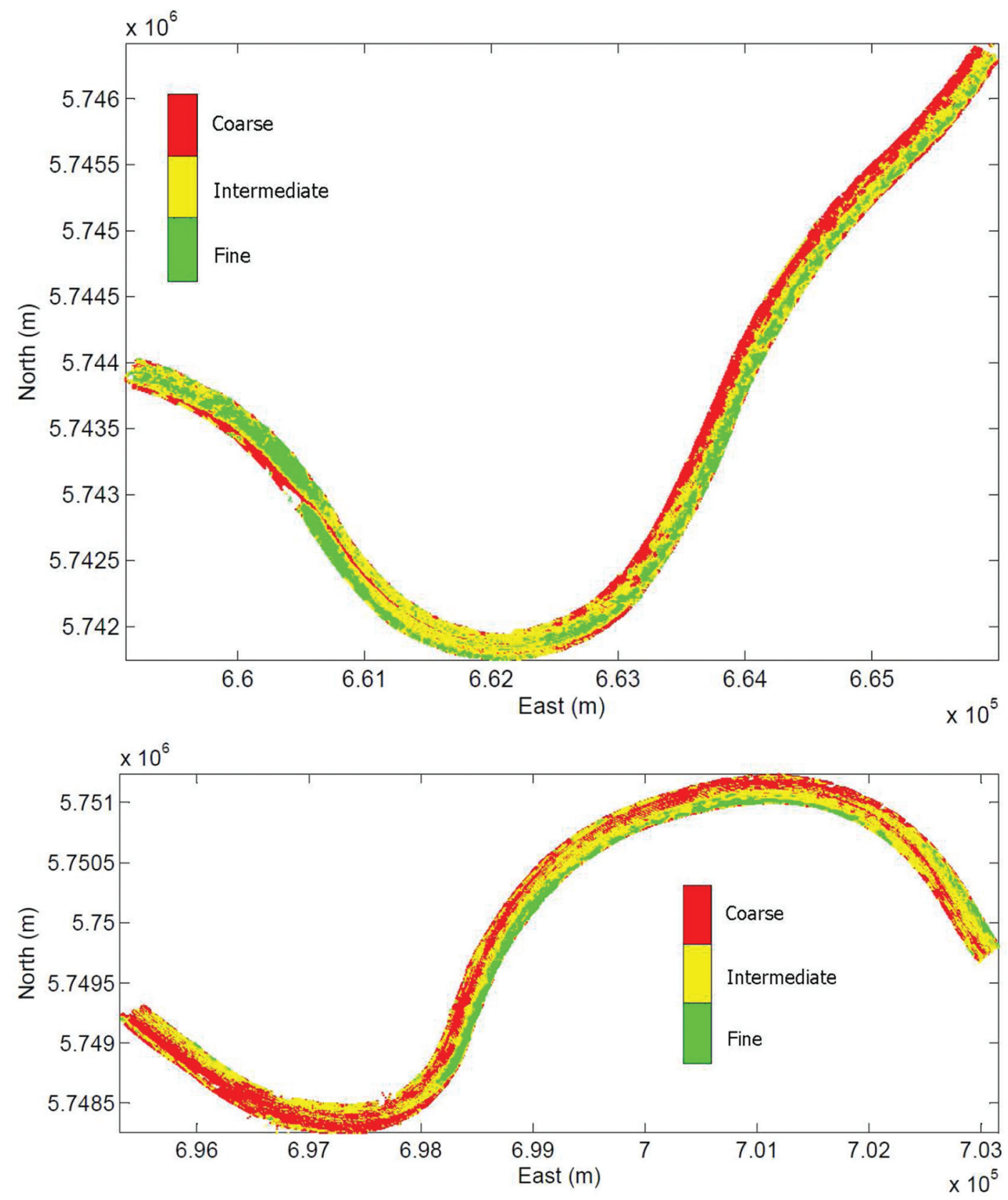

FIG. 7. Classification map based on the first two PCs and K-means methods applied to only the backscatter features in which the number of clusters was set to $k=3$; (top) Sint Andries and (bottom) Nijmegen.
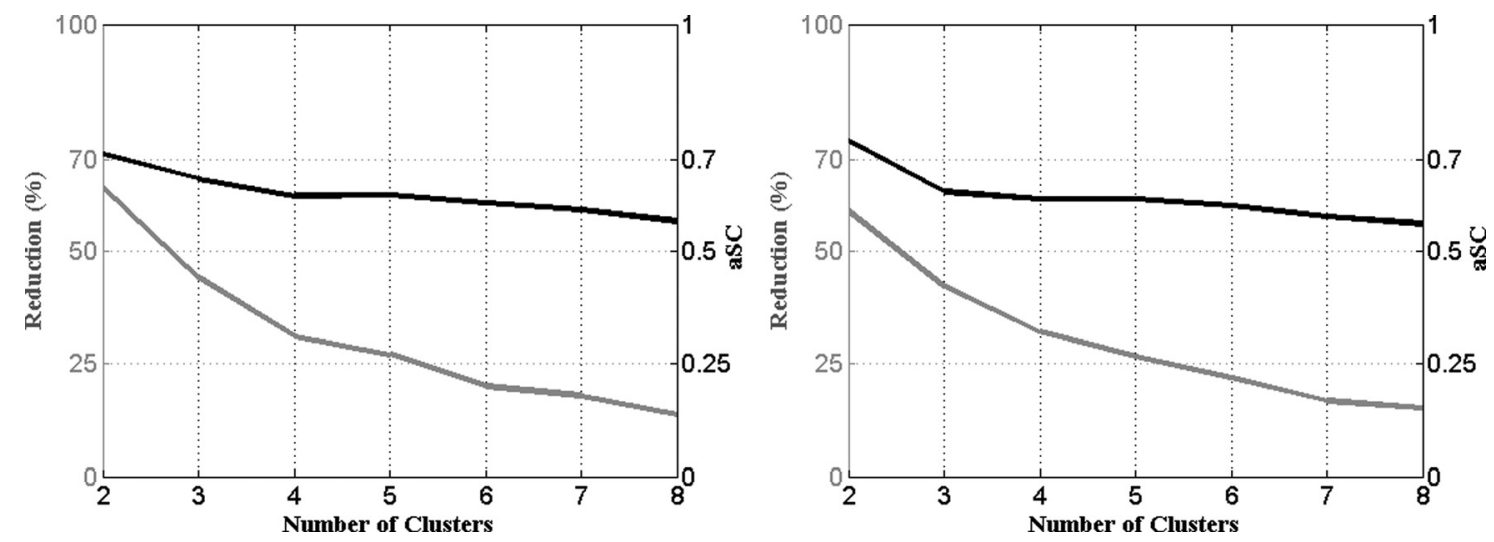

FIG. 8. Reduction percentage in the sum of distances (left $y$-axis — grey line) and average silhouette coefficient (right $y$-axis — black line) versus number of clusters (from 2 to 8 ) for (left) Sint Andries and (right) Nijmegen, when classifying using backscatter. 

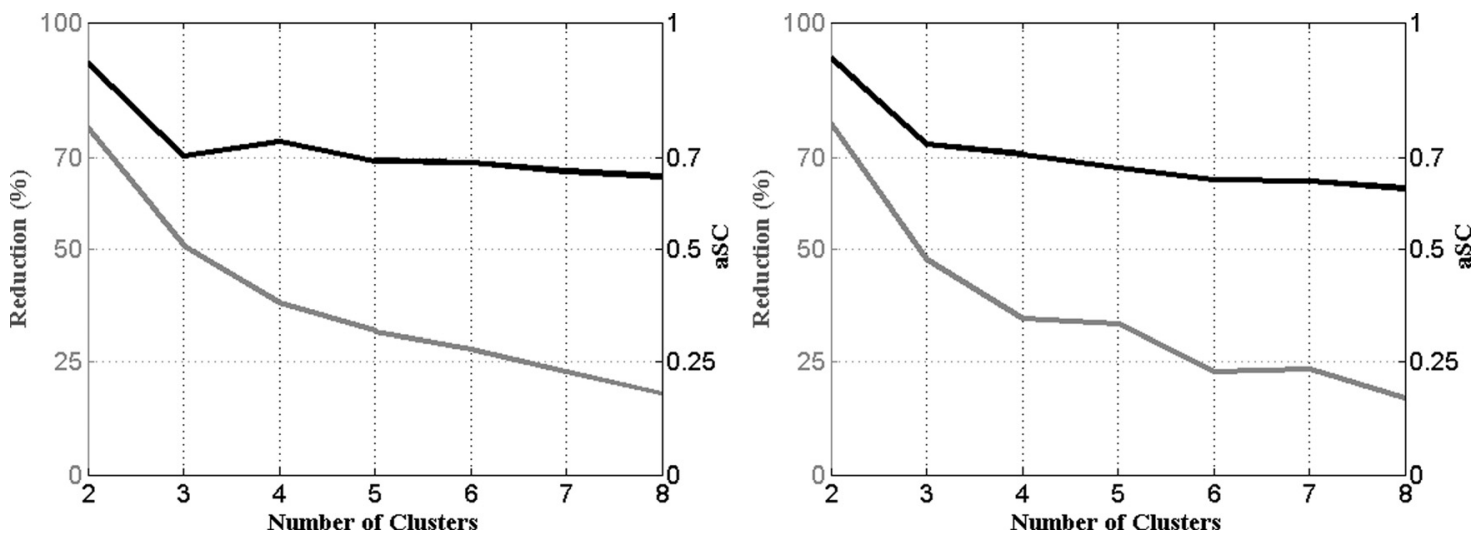

FIG. 9. Reduction percentage in the sum of distances (left y-axis — grey line) and average silhouette coefficient (right y-axis — black line) versus number of clusters (from 2 to 8) for (left) Sint Andries and (right) Nijmegen, when classifying using LS depth residuals.
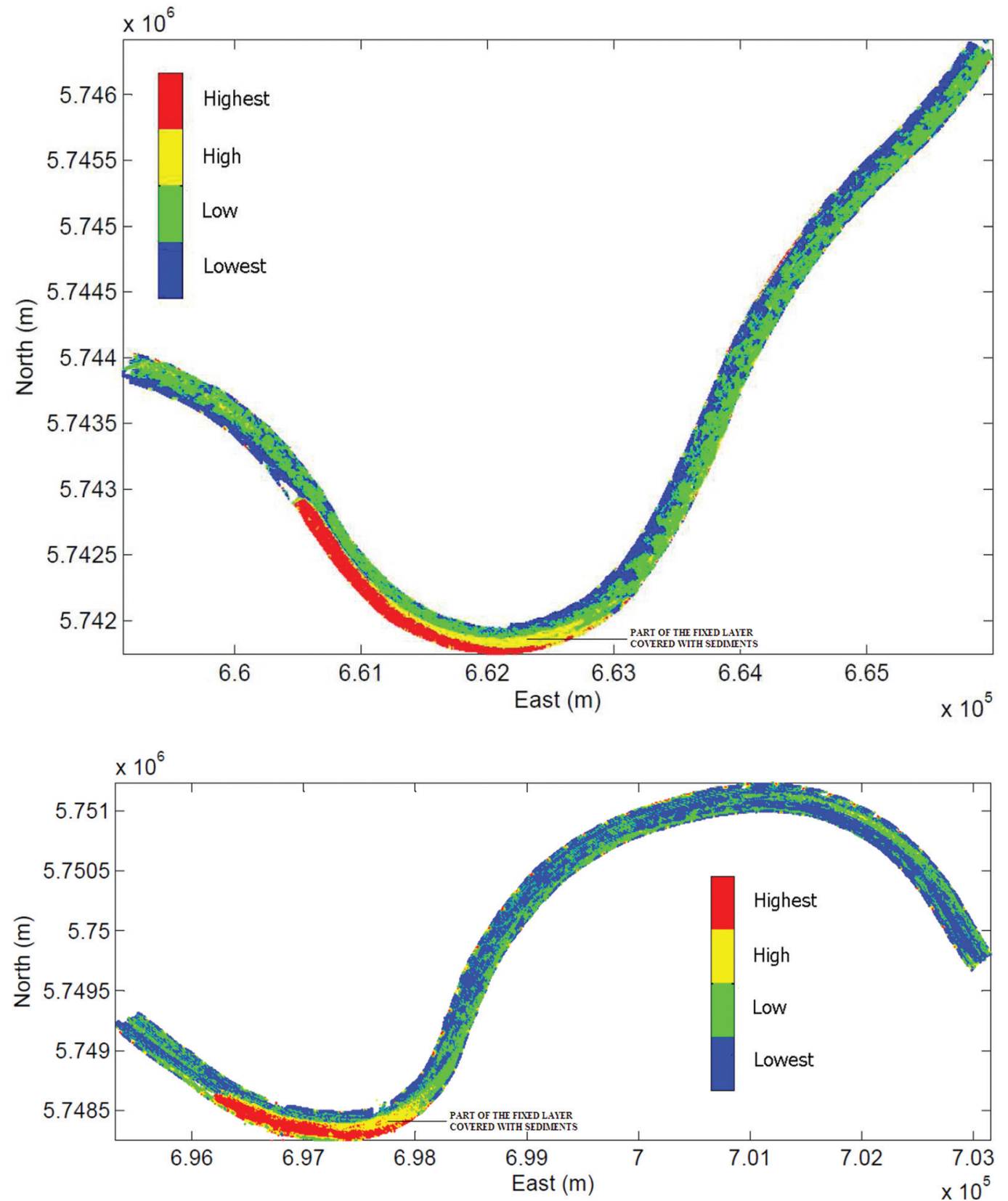

FIG. 10. Classification map in terms of degree of depth variations based on the first two PCs and K-means methods applied to only the depth residual features in which the number of clusters was set to $k=4$; (top) Sint Andries and (bottom) Nijmegen. 
very similar results can be obtained. The sediment distribution maps of Sint Andries and Nijmegen, are shown in Fig. 10. Here the first class (blue) has the lowest variations in depth residuals, indicating that the data points can best fit to the surface patch, and class 4 (red) has the highest variations in depth residuals.

We would intuitively expect that coarser sediment gives the highest variations (e.g., highest standard deviations). This however seems not to be the case, because highest variations belong to the finer sediment. That is likely due to the fact that in finer sediment "ripples" can be formed, while coarse sediments will usually form "dunes." Ripples, which are small triangular sand waves, usually are shorter than about $60 \mathrm{~cm}$ and not higher than about $60 \mathrm{~mm}$. Ripples typically being about 1 order of magnitude shorter than dunes. Also dunes generally form at larger flow and sediment transport rates, while ripples often form on the upstream slopes of dunes at smaller rates of flow.

The highest depth variations belong to the fixed layer, which is due to the riprap structure. It can therefore be clearly discriminated from the rest of the river. Moreover the northern part of the right-hand side of the fixed layer has been identified as a different class. We hypothesize that this part has been covered by sediments due to the river flow and the resulting sediment transport processes. This leads to lower depth variations with respect to the original fixed layer but higher variations compared to the entire river.

\section{Classification using all features}

This subsection considers all eight features presented in Table III. The objective of this subsection is (1) to further investigate the nature of all final features considered using the PCA process; (2) to assess the potential of using all features in order to identify the fixed layer; (3) to evaluate the maximum number of classes resulting from all features; (4) to apply this number of classes to both areas and make the classification maps; and (5) to correlate the classification results with the grab samples taken.

The four backscatter features are highly correlated to each other. This holds also for the four residuals features.
There is however no significant correlation among the backscatter features and the depth residual features; the average absolute correlation among them for the two areas is around 0.25 . This indicates that these two sources of information are independent to a large extent, and hence can provide complementary tools for classification. Therefore, any attempt for applying the PCA process on the combined features is expected to give the first two principal components as a combination of backscatter features and a combination of the residual features. This means that any pair of the features of the kind (BS, LS Residuals) can also be used for providing the classification map of the areas that is visually similar to what is presented in this subsection.

Again the PCA is used to combine the information provided from all the features. In Sint Andries, the first, second, and third PCs account for $68.9 \%, 29.4 \%$, and $1.2 \%$ of the variability of the data, respectively. These numbers change to $53.8 \%, 44.4 \%$, and $0.9 \%$ for Nijmegen. Together the first two PCs account for about $98 \%$ of the variability and hence will be used for further analysis. The results indicate that the first PC is influenced slightly more by the depth residual features than the backscatter features. The opposite holds for the second PC.

We set the number of classes to 4 based on the results of previous subsections. The first two PCs are fed to the $\mathrm{K}$-means clustering. Figure 11 presents the separation of the clusters versus the first and second PCs. A clear distinction between the clusters can be seen for both areas. The sediment distribution maps of Sint Andries and Nijmegen with a fixed number of four classes is given in Fig. 12. These results are in fact similar to those with three classes using only the backscatter features (Fig. 7) plus the fixed layer. The first three classes are mainly due to the backscatter effect. The fourth class is the fixed layer, which can only be detected if the LS depth residual features are used (cf. Figure 10 with four classes). This implies that the fixed layer has on average a similar backscatter property to the other parts of the river, but it definitely behaves differently on the LS depth residual features.

The next step is to assess the possibility of further discrimination. The combined plot of the $\%$ reduction in the
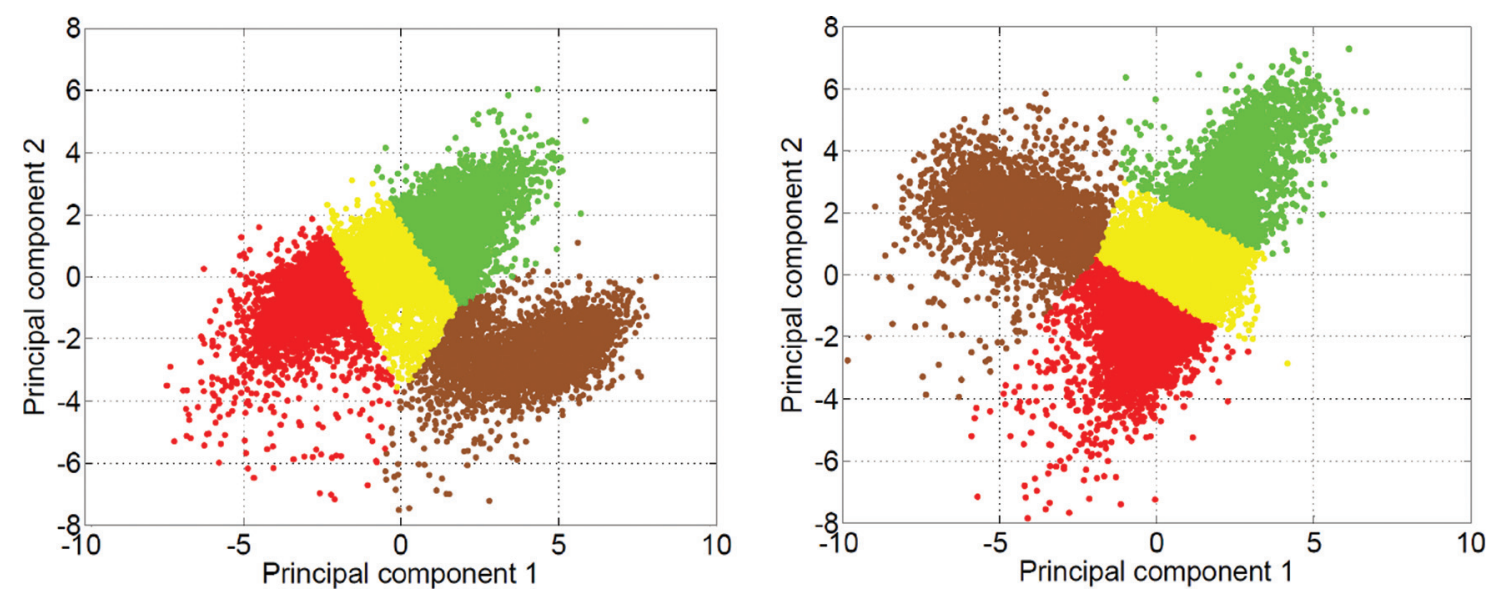

FIG. 11. Scatter plot of PC1 versus PC2 when K-means clustering is applied to the first two PCs obtained from all features of backscatter and depth residuals $(k=4)$; (left) Sint Andries and (right) Nijmegen. 

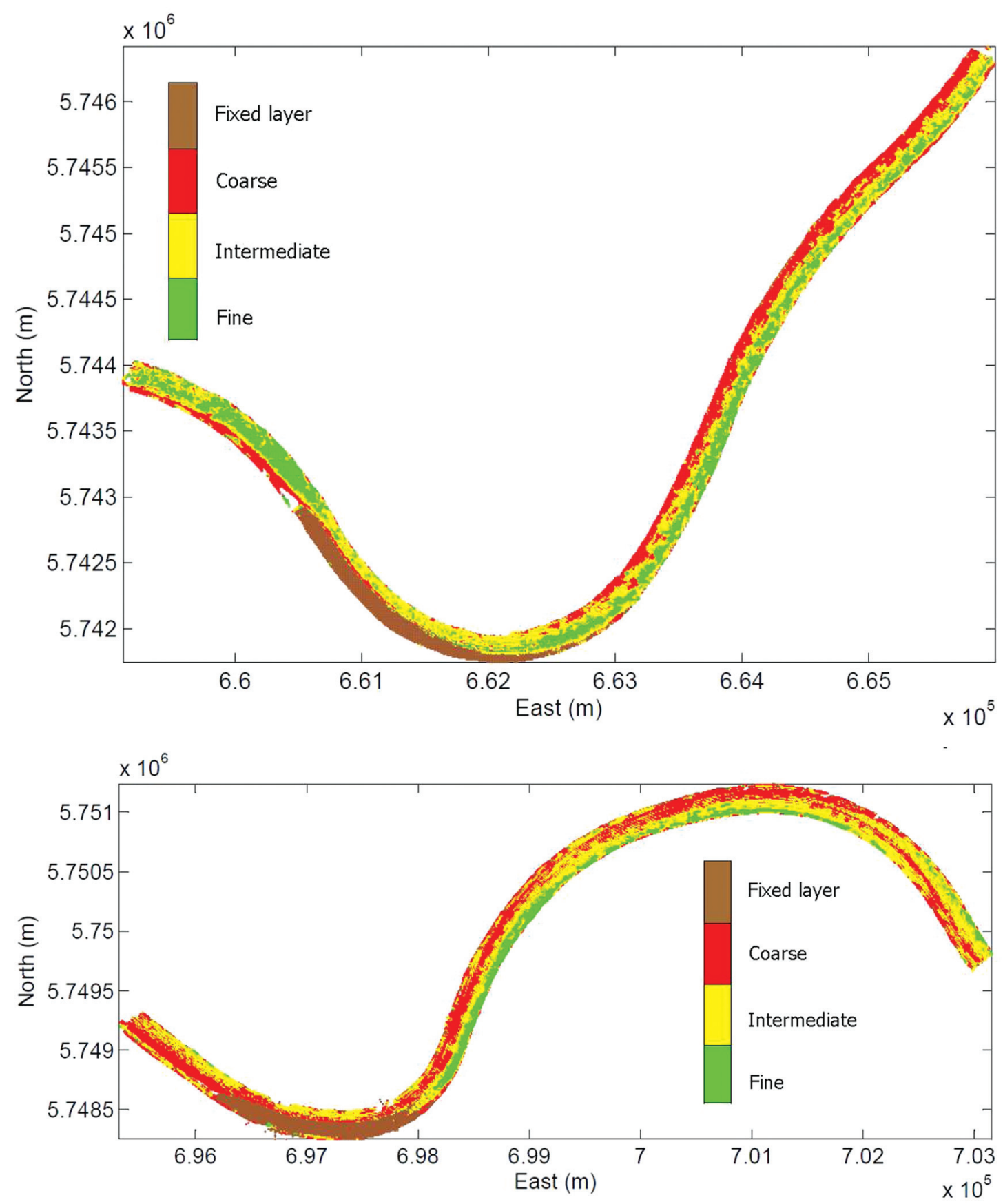

FIG. 12. Classification maps based on the first two PCs and K-means clustering when all features from backscatter and depth residuals are used $(k=4)$; (top) Sint Andries and (bottom) Nijmegen.

distances (left $y$-axis) and the average silhouette coefficient (right $y$-axis) against the number of clusters for Sint Andries (Fig. 13, left) and Nijmegen (Fig. 13, right) is given in Fig. 13. Although there is a drop for 5 clusters, there is another peak for 6 clusters before the values start to gradually drop with increasing clusters number. Therefore, the case of six clusters will be further investigated.

Figure 14 presents the separation of the clusters versus the first and second PCs for 6 classes. A clear distinction between the clusters can be seen for both areas. The resulting sediment distribution maps of Sint Andries and Nijmegen are shown in Fig. 15.
These results are in fact similar to those with four classes using only the backscatter features plus two fixed layers given by the depth residual features. Therefore, the first four classes are mainly due to the backscatter effect, and the fifth and sixth classes are mainly due to the depth residual features. This can also be seen in the scatter plots of the first and second PCs in Fig. 14. Classes 1 to 4 correspond to the finest to coarsest sediments respectively (as will become clear later in this section where the classification results are compared with the samples). The fixed layer can also be identified here. In addition, for both rivers, on the northern part of the right-hand side of the fixed layer, a separate class 

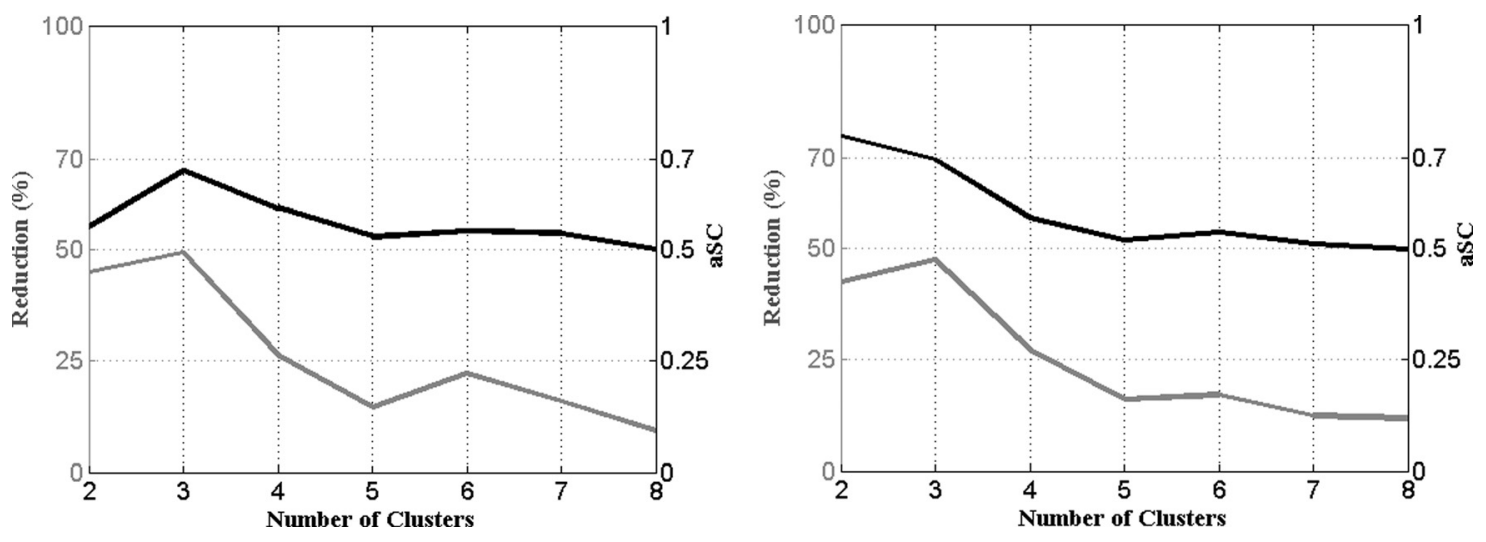

FIG. 13. Reduction percentage in the sum of distances (left y-axis — grey line) and average silhouette coefficient (right y-axis — black line) versus number of clusters (from 2 to 8) for (left) Sint Andries and (right) Nijmegen, when classifying using both backscatter and LS depth residuals.

can be identified. The degree of depth variations is lower compared with the original fixed layers and probably they (for both rivers) have been covered by sediments. This leads to lower depth variations with respect to the original fixed layer but higher variations compared to the entire river.

To assess the potential of converting the acoustic classes to riverfloor sediment properties such as mean grain size the classification results are compared with the grab samples.

To make the comparison easier we correlate the mean grain sizes to the acoustic classes 1, 2, 3, and 4; classes 5 and 6 were excluded because they belong to the fixed layer where no grab sample is available. Figure 16 shows the best linear fit, using the least squares method, between the classification results (classes) and the mean grain sizes expressed as $M_{z}=-\log _{2} d$ in phi units, where $d$ is the diameter of grain in millimeters. A sample is located in an area where surface patches of various classes are present. Each sample took the average class number of the surface patches within a radius of $10 \mathrm{~m}$ from it. The samples without any surface patches within this radius were not used for comparison. The corresponding estimated Pearson correlation coefficients are -0.84 and -0.71 for Sint Andries and Nijmegen, respectively. The same procedure was applied to the three first classes in Fig. 12. The correlation coefficients change to -0.84 and -0.75 for these two areas.

\section{Discussion of results}

The main contribution of this work is that it shows that the combination of features can provide insights to the sediment distribution on a riverbed otherwise hidden when only one of the features (backscatter or depth residuals) is used. The fixed layer is clearly visible and clearly highlighted in all maps, when the LS depth residuals or a combination of the LS depth residuals and backscatter were used. This however, is not the case when considering backscatter only. The inadequacy of detecting the fixed layer by using only the backscatter strength has raised many assumptions. ${ }^{31}$ The most probable explanation is that the blank areas between the stones and also a thin top layer of the fixed layer is filled with sand, due to transport processes in the outer bend of the rivers, up to a point that this finer layer dominates the discriminative performance of the sonar. By combining backscatter and bathymetry information it was possible to preserve the main patterns of the classification with 3 classes provided by the backscatter but also adds one more class: the fixed layer.
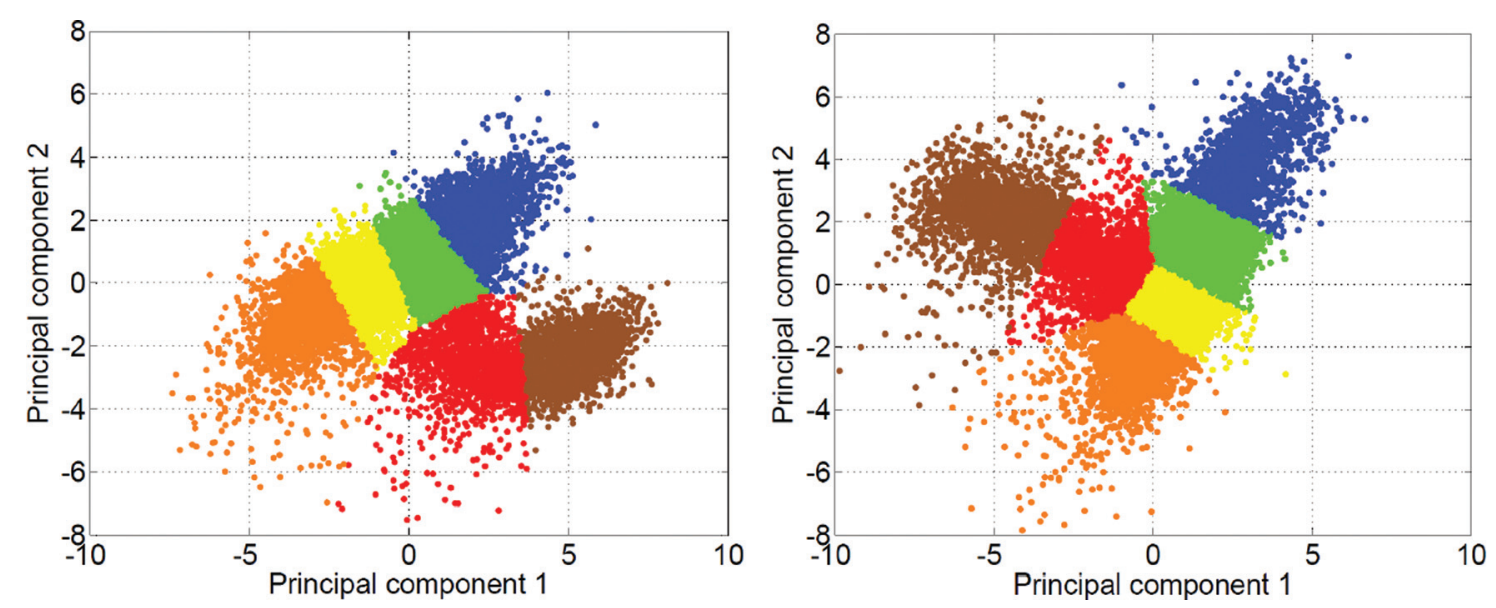

FIG. 14. Scatter plot of PC1 versus PC2 when K-means clustering is applied to the first two PCs obtained from all features of backscatter and depth residuals $(k=6)$; (left) Sint Andries and (right) Nijmegen. 

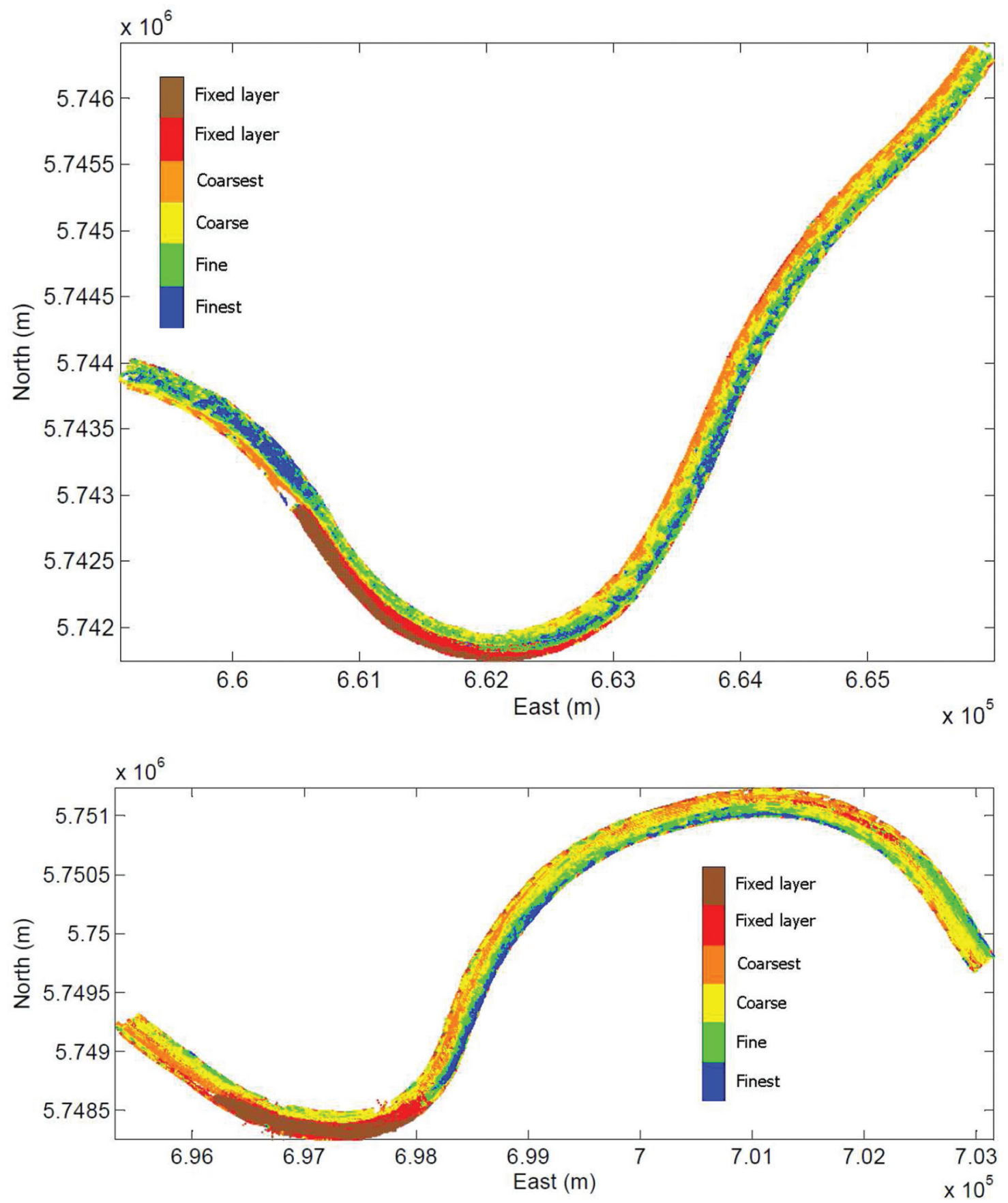

FIG. 15. Classification maps based on the first two PCs and K-means clustering when all features from backscatter and depth residuals are used ( $k=6$ ); (top) Sint Andries and (bottom) Nijmegen.

The depth residual features and the combination of all features gave rise to possible artifacts in the maps. Small areas, at the borders of the river have been classified with the same color as the fixed layer (brown). This is hypothesized to be the effect of scour holes, resulting in high backscatter values and irregular bathymetry. Because the standard deviations of the depth residuals were larger in the finer sediment than the coarser sediment, we hypothesize that this is due to the riverbed ripples.

The final features used were a combination of 4 backscatter and 4 residual features. Though the final results presented were based on all 8 features, the high correlation among the backscatter features as well as the high correla- tion among the depth residual features suggested that one can obtain very similar results if for example use is made of only the mean backscatter and the standard deviation of the depth residual.

\section{SUMMARY AND CONCLUSIONS}

In this paper, PCA and K-means clustering were used for the sediment classification of two parts with similar characteristics (groynes, fixed layer) of the river Waal, in the Netherlands. Three cases were investigated: (1) classification with only the backscatter features, (2) classification with only the LS depth residual features, and (3) classification 

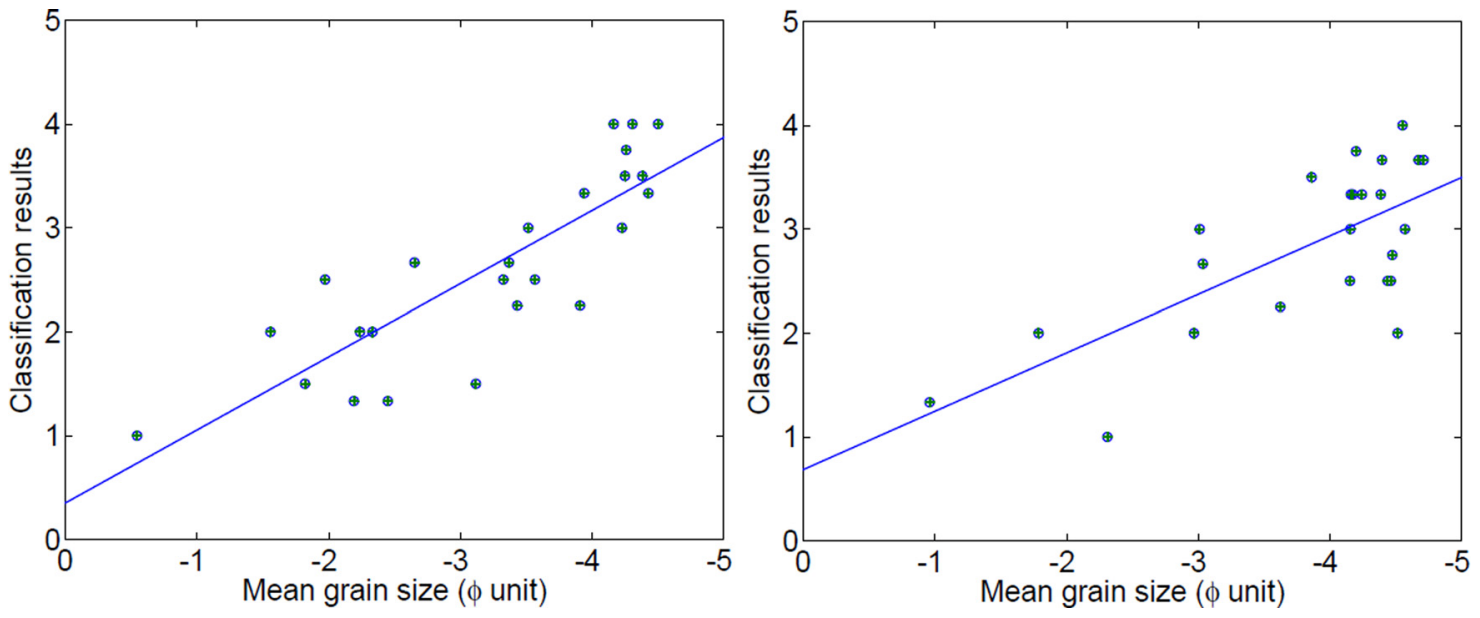

FIG. 16. (Color online) Mean grain size of individual samples versus classification results of Fig. 15, where class numbers 1, 2, 3, 4 belong to the finest, fine, coarse, and coarsest sediment; (left) Sint Andries and (right) Nijmegen.

with all backscatter and LS depth residual features. In the first case, the results were compared with previous work, ${ }^{16,17}$ where a Bayesian classification methodology was used for the classification process. The results between the two methods are similar: three classes seem to be appropriate for both methods for these particular areas. Deviations in the classification results can be attributed to the different averaging procedures. When averaging, a limited number of beams was used in classification in Ref. 16 due to the angular dependence, whereas in the present contribution, the averaging was performed over a much larger number of measurement, which was made possible due to the standardization. For the second case, use was made of the LS depth residual features only. It was shown that these features can clearly discriminate between the fixed layer and the remainder of the area. The third case, with all features, could even further discriminate within the fixed layer and within the sediment classes; six classes in total were included. The conclusion here is that the fixed layer can be detected for both areas when use is made of the depth residual features. Using only the backscatter one cannot classify the fixed layer. The following aspects of the research can also be highlighted.

(1) The depth residual features could detect further than only the fixed layer. Other bottom structures such as the fixed layer covered by sediments and the riverbed ripples can also be identified.

(2) Due to the high correlation among the features, one can conclude that the combination of only one backscatter feature with only one depth residual feature can provide results that are very similar to those obtained based on all the features.

(3) Significant correlation coefficient between the classification results and the mean grain sizes along with the significant slope of the best linear fit (Fig. 16) indicate high potential capability of the proposed method for riverbed sediment classification.

\section{ACKNOWLEDGMENTS}

The research is financially supported by the Dutch Ministry of Transportation and Water Management, Rijkswater- staat. We acknowledge in particular Dr. Arjan Sieben, Ir. Ben Dierikx, and Adri Wagener for providing the Waal data and for their support during the project. Special thanks also to Dr. Erik Mosselman from Deltares for his valuable contribution.

${ }^{1}$ M. M. Harris, W. E. Avera, A. Abelev, F. W. Bentrem, and L. D. Bibee, "Sensing shallow seafloor and sediment properties, recent history," Proc. OCEANS 2008 MTS/IEEE, Quebec, ON, 1-11 (2008).

${ }^{2} \mathrm{P}$. Blondel and O. Gomez Sichi, "Textural analyses of multibeam sonar imagery from Stanton Banks, Northern Ireland continental shelf," Appl. Acoust., 70, 1288-1297 (2009).

${ }^{3}$ J. M. Augustin, R. Le Suave, X. Lurton, M. Voisset, S. Dugelay, and C. Satra, "Contribution of the multibeam acoustic imagery to the exploration of the sea bottom," Mar. Geophys. Res. 18(2-4), 459-486 (1996).

${ }^{4}$ B. Chakraborty, H. W. Schenke, V. Kodagali, and R. Hagen, "Seabottom characterization using multibeam echosounder angular backscatter: An application of the composite roughness theory," IEEE Trans. Geosci. Remote Sensing, 38(5), 2419-2422 (2000).

${ }^{5}$ J. M. Preston, "Automated acoustic seabed classification of multibeam images of Stanton Banks,” Appl. Acoust. 70(10), 1277-1287 (2009).

${ }^{6}$ J. M. Preston, A. C. Christney, L. S. Beran, and W. T. Collins, "Statistical seabed segmentation-From images and echoes to objective clustering," in Proceedings of the Seventh European Conference on Underwater Acoustics, Delft, Netherlands (2004), pp. 813-818.

${ }^{7}$ J. M. Preston, D. R. Parrot, and W. T. Collins, "Sediment classification based on repetitive multibeam bathymetry survey of an offshore disposal site," in Proceedings IEEE OCEANS 2003, San Diego, CA (22-26 September 2003), pp. 69-75, Vol. 9.

${ }^{8}$ W. T. Collins, and J. M. Preston, "Multibeam seabed classification," Int. Ocean Syst. 6(4), 12-15 (2002).

${ }^{9}$ J. L. Galloway, "Systematic acoustic seafloor habitat mapping of the British Columbia coast," in Marine Habitat Mapping Technology for Alaska, edited by J. R. Reynolds and H. G. Greene (2008), pp. 195-201.

${ }^{10}$ C. J. Brown, B. J. Todd, V. E. Kostylev, and R. A. Pickrill, "Image-based classification of multibeam sonar backscatter data for objective surficial sediment mapping of Georges Bank, Canada," Cont. Self Res. 31(2), 100-119 (2011)

${ }^{11}$ C. McGonigle, C. Brown, R. Quinn, and J. Grabowski, "Evaluation of image-based multibeam sonar backscatter classification for benthic habitat discrimination and mapping at Stanton Banks, UK," Estuarine Coastal Shelf Sci. 81(3), 423-437 (2009).

${ }^{12}$ L. Atallah and P. Probert Smith, "How useful is bathymetry information in the classification of high frequency sonar surveys?," in Proceedings of the International Conference "Underwater Acoutic Measurements: Technologies and Results," Heraklion, Crete, Greece, 2005, available at http:// www.doc.ic.ac.uk/ latallah/bathy_uam.pdf (Last viewed September 6, 2011). 
${ }^{13}$ G. R. Cutter, Jr., Y. Rzhanov, and L. A. Mayer, "Automated segmentation of seafloor bathymetry from multibeam echosounder data using local Fourier histogram texture fixtures," J. Exp. Mar. Biol. Ecol. 285-286, 355-370 (2003).

${ }^{14}$ I. Marsh and C. Brown, "Neural classification of multibeam backscatter and bathymetry data from Stanton Bank (Area IV)," Appl. Acoust. 70(10), 1269-1276 (2009).

${ }^{15}$ C. J. Brown, S. J. Smith, P. Lawton, and J. T. Anderson, "Benthic habitat mapping: A review of progress towards improved understanding of the spatial ecology of the seafloor using acoustic techniques," Estuarine Coastal Shelf Sci. 92, 502-520 (2011).

${ }^{16}$ A. R. Amiri-Simkooei, M. Snellen, and D. G. Simons, "Riverbed sediment classification using multi-beam echo-sounder backscatter data," J. Acoust. Soc. Am. 126, 1724-1738 (2009).

${ }^{17}$ A. R. Amiri-Simkooei, M. Snellen, and D. G. Simons, "Using multi-beam echo-sounder backscatter data for sediment classification in very shallow water environment," in Proceedings of Underwater Acoustic Measurements: Technologies and Results, Nafplion, Greece, 21-26 June (2009), available at http://promitheas.iacm.forth.gr/uam2009/lectures/pdf/29-1.pdf (Last viewed September 6, 2011).

${ }^{18}$ C. J. Sloff, E. Mosselman, and J. Sieben, "Effective use of non-erodible layers for improving navigability," in Proceedings of River Flow 2006, Lisbon, 6-8 September, edited by R. M. L. Ferreira, E. C. T. L. Alves, J. G. A. B. Leal, and A. H. Cardoso, Taylor and Francis, London (2006), pp. 1211-1220, Vol. 2.

${ }^{19}$ E. Hammerstad, "Backscattering and seabed image reflectivity," EM Technical Note, (2000), available at http://www.km.kongsberg.com/ks/ web/nokbg0397.nsf/AllWeb/226C1AFA658B1343C1256D4E002EC764/ \$file/EM_technical_note_web_BackscatteringSeabedImageReflectivity. pdf? OpenElement (Last viewed September 6, 2011).

${ }^{20}$ D. G. Simons, and M. Snellen, "A Bayesian approach to seafloor classification using multi-beam echo-sounder backscatter data," Appl. Acoust. 70, 1258-1268 (2009).
${ }^{21} \mathrm{~K}$. Pearson, "On lines and planes of closest fit to systems of points in space," Philos. Mag. 2(6), 559-572 (1901).

${ }^{22} \mathrm{H}$. Hotelling, "Analysis of a complex of statistical variables into principal components,” J. Educ. Psychol. 24(7), 498-520 (1933).

${ }^{23}$ B. F. J. Manly, Multivariate statistical methods, A Primer, 2nd ed. (Chapman and Hall, London, 1994), Chap. 6, pp. 77-79.

${ }^{24}$ R. A. Johnson and D. W. Wicherm, Applied Multivariate Statistical Analysis, 5th ed. (Prentice Hall, Englewood Cliffs, NJ, 2002), Chap. 8, pp. 426-462.

${ }^{25}$ P. R. Peres-Neto, D. A. Jackson, and K. M. Somers, "How many principal components? Stopping rules for determining the number of non-trivial axes revisited," Comput. Stat. Data Anal., 49, 974-997 (2005).

${ }^{26}$ I. T. Jolliffe, Principal Component Analysis, Springer Series in Statistics, 2nd ed. (Springer, New York, 2002), Chap. 6, pp. 111-149.

${ }^{27} \mathrm{~J}$. McQuenn, "Some methods for classification and analysis of multivariate observations," in Proceedings of 5th Berkeley Symposium on Mathematical Statistics and Probability (University of California Press, Berkeley, 1967), pp. 281-297.

${ }^{28}$ G. A. F. Seber, Multivariate Observations, Series in Probability and Mathematical Statistics (John Wiley and Sons, Hoboken, NJ, 1984), Chap. 7, pp. 379-386.

${ }^{29}$ L. Kaufman, and P. J. Rousseeuw, Finding Groups in Data, An Introduction to Cluster Analysis, Wiley Series in Probability and Mathematics (John Wiley and Sons, Hoboken, NJ, 1990), Chap. 2, pp. 83-88.

${ }^{30}$ P. J. G. Teunissen, D. G. Simons, and C. C. J. M. Tiberius, Probability and Observation Theory (Department of Earth Observation and Space Systems (DEOS), Faculty of Aerospace Engineering, Delft University of Technology, Delft, Netherlands, 2005), Chap. 3, pp. 97-161.

${ }^{31}$ D. Eleftherakis, E. Mosselman, A. R. Amiri-Simkooei, S. Giri, M. Snellen, and D. G. Simons, "Identifying changes in river bed morphology and bed sediment composition using multi-beam echo-sounder measurements," in Proceedings of the 10th European conference on Underwater Acoustics, Istanbul, Turkey (5-9 July, 2010), pp. 1365-1373. 